\title{
AGILE DETECTION OF A STRONG GAMMA-RAY FLARE FROM THE BLAZAR 3C 454.3
}

\author{
S. Vercellone, ${ }^{1,2}$ A. W. Chen,,${ }^{1,3}$ A. Giuliani, ${ }^{1}$ A. Bulgarelli, ${ }^{4}$ I. Donnarumma, ${ }^{5}$ I. Lapshov, ${ }^{5}$ M. Tavani,, 6 A. Argan, ${ }^{5}$ \\ G. Barbiellini, ${ }^{7}$ P. Caraveo, ${ }^{1}$ V. Cocco,${ }^{5}$ E. Costa,${ }^{5}$ F. D’Ammando, ${ }^{5,6}$ E. Del Monte, ${ }^{5}$ G. De Paris, ${ }^{5}$ G. Di Cocco, ${ }^{4}$ \\ Y. Evangelista, ${ }^{5}$ M. Feroci, ${ }^{5}$ M. Fiorini, ${ }^{1}$ T. Froysland,${ }^{3,6}$ F. Fuschino, ${ }^{4}$ M. Galli, ${ }^{8}$ F. Gianotti, ${ }^{4}$ C. Labanti, ${ }^{4}$ \\ F. Lazzarotto,${ }^{5}$ P. Lipari, ${ }^{9}$ F. Longo,${ }^{7}$ M. Marisaldi, ${ }^{4}$ F. Mauri, ${ }^{10}$ S. Mereghetti, ${ }^{1}$ A. Morselli, ${ }^{11}$ L. Pacciani, ${ }^{5}$ \\ A. Pellizzoni, ${ }^{1}$ F. Perotti, ${ }^{1}$ P. Picozza, ${ }^{11}$ M. Prest, ${ }^{12}$ G. Pucella, ${ }^{5}$ M. Rapisarda, ${ }^{13}$ P. Soffitta, ${ }^{5}$ M. Trifoglio, ${ }^{4}$ \\ A. Trois,${ }^{5}$ E. Vallazza, ${ }^{7}$ V. Vittorini, ${ }^{6}$ A. Zambra, ${ }^{1}$ D. Zanello, ${ }^{9}$ C. Pittori,${ }^{14}$ F. Verrecchia, ${ }^{14}$ D. Gasparrini, ${ }^{14}$ \\ S. Cutini, ${ }^{14}$ P. Giommi, ${ }^{14}$ L. A. Antonelli, ${ }^{14}$ S. Colafrancesco, ${ }^{14}$ and L. Salotti ${ }^{15}$ \\ Received 2007 December 12; accepted 2008 February 8; published 2008 March 3
}

\section{ABSTRACT}

We report the first blazar detection by AGILE. AGILE detected 3C 454.3 during a period of strongly enhanced optical emission in 2007 July. AGILE observed the source with a dedicated repointing during the period 2007 July 24-30 with its two co-aligned imagers, the Gamma-Ray Imaging Detector and the hard X-ray imager SuperAGILE sensitive in the $30 \mathrm{MeV}$ to $50 \mathrm{GeV}$ and 18-60 keV ranges, respectively. Over the entire period, AGILE detected $\gamma$-ray emission from 3C 454.3 at a significance level of $13.8 \sigma$ with an average flux $(E>100 \mathrm{MeV})$ of $(280 \pm 40) \times 10^{-8}$ photons $\mathrm{cm}^{-2} \mathrm{~s}^{-1}$. The $\gamma$-ray flux appears to be variable toward the end of the observation. No emission was detected by Super-AGILE in the energy range $20-60 \mathrm{keV}$, with a $3 \sigma$ upper limit of $2.3 \times$ $10^{-3}$ photons $\mathrm{cm}^{-2} \mathrm{~s}^{-1}$. The $\gamma$-ray flux level of $3 \mathrm{C} 454.3$ detected by AGILE is the highest ever detected for this quasar and among the most intense $\gamma$-ray fluxes ever detected from flat-spectrum radio quasars.

Subject headings: gamma rays: observations - quasars: individual (3C 454.3)

\section{INTRODUCTION}

Among active galactic nuclei (AGNs), blazars show strong flux variability at almost all frequencies of their spectral energy distributions (SEDs). The Energetic Gamma Ray Experiment Telescope (EGRET) instrument on board the Compton GammaRay Observatory (CGRO) detected, above $30 \mathrm{MeV}$, several AGNs, establishing the blazars as a class of $\gamma$-ray sources (Hartman et al. 1999). Gamma-ray blazars are characterized by high variability on different timescales. At energies above 30 $\mathrm{MeV}$, variability has been detected on timescales from 1 day (e.g., PKS 1622-297; Zhang et al. 2002) to 1 month (e.g., PKS 0208-512; von Montigny et al. 1995).

The flat-spectrum radio quasar (FSRQ) 3C 454.3 (PKS $2251+158 ; z=0.859)$ was detected by EGRET in 1992 during an intense $\gamma$-ray flaring episode (Hartman et al. 1992, 1993) when the blazar flux $(E>100 \mathrm{MeV})$ was observed to vary within the range (0.4-1.4) $\times 10^{-6}$ photons $\mathrm{cm}^{-2} \mathrm{~s}^{-1}$. In 1995, a 2 week EGRET campaign detected a $\gamma$-ray flux $<1 / 5$ of its historical maximum (Aller et al. 1997). Vercellone et al. (2004), analyzing the 67 EGRET $\gamma$-ray blazars by defining an activity index $\psi$,

\footnotetext{
${ }^{1}$ INAF/IASF-Milano, Via E. Bassini 15, I-20133 Milan, Italy.

${ }^{2}$ Corresponding author; stefano@iasf-milano.inaf.it.

${ }^{3}$ CIFS-Torino, Viale Settimio Severo 3, I-10133 Torino, Italy.

${ }^{4}$ INAF/IASF-Bologna, Via Gobetti 101, I-40129 Bologna, Italy.

${ }^{5}$ INAF/IASF-Roma, Via del Fosso del Cavaliere 100, I-00133 Rome, Italy.

${ }^{6}$ Dipartimento di Fisica, University “Tor Vergata," Via della Ricerca Scientifica 1, I-00133 Rome, Italy.

${ }^{7}$ Dipartimento di Fisica and INFN, Via Valerio 2, I-34127 Trieste, Italy.

${ }^{8}$ ENEA-Bologna, Via Biancafarina 2521, I-40059 Medicica (BO), Italy.

9 INFN-Roma "La Sapienza," Piazzale A. Moro 2, I-00185 Rome, Italy.

${ }^{10}$ INFN-Pavia, Via Bassi 6, I-27100 Pavia, Italy.

${ }^{11}$ INFN-Roma "Tor Vergata," Via della Ricerca Scientifica 1, I-00133 Rome, Italy

${ }^{12}$ Dipartimento di Fisica, University dell'Insubria, Via Valleggio 11, I-22100 Como, Italy.

${ }^{13}$ ENEA-Roma, Via E. Fermi 45, I-00044 Frascati (Rome), Italy.

${ }^{14}$ ASI-ASDC, Via G. Galilei, I-00044 Frascati (Rome), Italy.

15 ASI, Viale Liegi 26, I-00198 Rome, Italy.
}

singled out $3 \mathrm{C} 454.3$ as the source with the highest activity index $\left(\psi=0.033 \times 10^{-7} \mathrm{~cm}^{-2} \mathrm{~s}^{-1}\right)$ among EGRET blazars.

In 2005, 3C 454.3 displayed major flaring activity in almost all energy bands (see Giommi et al. 2006). In the optical, it reached $R=12.0 \mathrm{mag}$ (Villata et al. 2006), and it was detected by INTEGRAL at a flux ${ }^{16}$ level of $\sim 3 \times 10^{-2}$ photons $\mathrm{cm}^{-2}$ $\mathrm{s}^{-1}$ in the 3-200 keV energy band (Pian et al. 2006). The radio flux began increasing right after the optical flare and reached a maximum about a year later (Villata et al. 2007). Unfortunately, at that time no high $\gamma$-ray instrument was operational. As pointed out by Pian et al. (2006) simultaneous observations of FSRQs by means of high-energy instruments are crucial to precisely locate the inverse Compton peak and to constrain the physical mechanisms operating in AGN flares.

In 2007 July, 3C 454.3 showed significant activity with strong optical flaring episodes (G. Tosti 2007, private communication), reaching $R=12.8$ mag. ${ }^{17}$ This time, the recently launched AGILE was performing its Science Performance Verification Phase (SVP) and devoted a week to the observation of $3 \mathrm{C} 454.3$, joining a multifrequency campaign reacting to the enhanced optical activity.

AGILE, a mission (Tavani et al. 2006) of the Italian Space Agency (ASI) devoted to high-energy astrophysics, is currently the only space mission capable of observing cosmic sources simultaneously in the energy bands $18-60 \mathrm{keV}$ and $30 \mathrm{MeV}$ to $50 \mathrm{GeV}$. It was launched on 2007 April 23 by the Indian PSLV-C8 rocket from the Satish Dhawan Space Center SHAR, Sriharikota. The AGILE equatorial orbit (average height 540 $\mathrm{km}$, inclination angle $2.5^{\circ}$ ) is ideal for high-energy observations because of its low-background environment. After the postlaunch Commissioning Phase (2007 May-June), AGILE started its SVP.

In this Letter we present an analysis of the AGILE data obtained during the 3C 454.3 observations. Preliminary results

\footnotetext{
${ }^{16}$ Assuming a Crab-like spectrum.

${ }^{17}$ See http://users.utu.fi/ kani/1m/index.html.
} 
were communicated in Vercellone et al. (2007) and Bulgarelli et al. (2007). The results of a multiwavelength campaign on 3C 454.3 and the theoretical modeling will be presented in a forthcoming paper. Throughout this Letter the quoted uncertainties are given at the $1 \sigma$ level, unless otherwise stated.

\section{THE AGILE INSTRUMENT}

AGILE (M. Tavani et al. 2008a, in preparation) has a threeaxis attitude stabilization with fixed solar panels. Because of the solar panel constraints, AGILE must maintain the instrument axis at $90^{\circ}$ orientation from the Sun at all times. Thus, the locus of allowed pointings forms a great circle in the sky. The AGILE scientific instrument (M. Tavani et al. 2008b, in preparation) is very compact and combines four active detectors yielding broadband coverage from hard X-rays to $\gamma$-rays. Gamma-ray detection is obtained by the combination of the Silicon Tracker, the Mini-Calorimeter, and the Anti-Coincidence System; these three detectors form the AGILE GammaRay Imaging Detector (GRID). The Silicon Tracker (Prest et al. 2003; Barbiellini et al. 2001) and the on-board trigger logic (A. Argan et al. 2008, in preparation) are optimized for $\gamma$-ray imaging in the $30 \mathrm{MeV}$ to $50 \mathrm{GeV}$ energy band. A nonimaging CsI detector (Mini-Calorimeter, MCAL) is positioned under the Silicon Tracker and is sensitive in the $0.4-100 \mathrm{MeV}$ energy band (Labanti et al. 2006). A co-aligned coded-mask hard Xray imager (SA; Feroci et al. 2007; Costa et al. 2001) ensures simultaneous coverage in the $18-60 \mathrm{keV}$ energy band. A segmented Anti-Coincidence System (ACS) made of a plastic shield surrounds all active detectors (Perotti et al. 2006). Table 1 provides the relevant parameters for both AGILE imaging detectors (AGILE-GRID and Super-AGILE) as computed on the basis of on ground calibrations, GRID beam tests, and extensive Monte Carlo simulations.

A crucial part of the GRID event processing is provided by the on-board Data Handling unit (Giuliani et al. 2006; A. Argan et al. 2008, in preparation). The on-ground data processing handles the GRID and Super-AGILE data through dedicated software developed by the instrument teams and integrated into an automatic pipeline system at the ASI Science Data Center $\left(\mathrm{ASDC}^{18}\right)$. The reduction software runs both at the ASDC and at the Instrument Institutes.

The SVP is aimed at testing and optimizing the overall scientific performance of the instrument, including in-flight calibrations. At the time of writing, the SVP has just been completed and only a partial analysis of the in-flight calibration data is available; therefore we restrict our analysis to the $E>100 \mathrm{MeV}$ energy range. However, the successful observations and tests carried out during the Commissioning Phase allow us to reliably process the data and obtain preliminary scientific results.

\section{AGILE OBSERVATION OF 3C 454.3}

At the epoch of the 3C 454.3 optical flare (2007 July 1921) the closest pointing position allowed by the solar panel constraints ranged within $35^{\circ}-40^{\circ}$ from the source. Because of the remarkably large $(\sim 3 \mathrm{sr})$ field of view (FOV) of the GRID and the successful detection of the Vela pulsar at $\sim 55^{\circ}$ off-axis obtained during the SVP, AGILE could reliably study 3C 454.3 despite its large off-axis position. The observation were performed between 2007 July 24 14:30 UT and 2007 July 30 11: $40 \mathrm{UT}$, for a total pointing duration of $\sim 5.8$ days.
TABLE 1

Gamma-Ray Imaging Detector and Super-AGILE Characteristics

\begin{tabular}{|c|c|c|}
\hline Parameter & AGILE-GRID & Super-AGILE \\
\hline Energy range & $30 \mathrm{MeV}-50 \mathrm{GeV}$ & $18-60 \mathrm{keV}$ \\
\hline Field of view & $\sim 3 \mathrm{sr}$ & $2 \times\left(107^{\circ} \times 68^{\circ}\right)^{\mathrm{a}}$ \\
\hline Sensitivity & $\begin{array}{l}3 \times 10^{-7} \text { photons } \\
\mathrm{cm}^{-2} \mathrm{~s}^{-1 \mathrm{~b}}\end{array}$ & $\sim 15 \mathrm{mcrab}^{\mathrm{c}}$ \\
\hline Angular resolution & $1.2^{\circ \mathrm{d}}$ & $6^{\prime}$ e \\
\hline Source location accuracy & $\sim 15^{\prime \mathrm{f}}$ & $\sim 2^{\prime}-3^{\prime} \mathrm{g}$ \\
\hline Energy resolution & $\Delta E / E \sim 1^{\mathrm{h}}$ & $\Delta E=8 \mathrm{keV}^{\mathrm{i}}$ \\
\hline Absolute time resolution & $\sim 2 \mu \mathrm{s}$ & $\sim 5 \mu \mathrm{s}$ \\
\hline $\begin{array}{l}{ }^{\mathrm{a}} \mathrm{FOV} \text { of each half-det } \\
{ }^{\mathrm{b}} E>100 \mathrm{MeV}, 5 \sigma \text { in } \\
{ }^{\mathrm{c}} 5 \sigma \text { in } 50 \mathrm{ks} \text {, on-axis. } \\
{ }^{\mathrm{d}} 68 \% \text { containment radi } \\
{ }^{\mathrm{e}} \mathrm{Sky} \text { pixel size on-axis } \\
{ }^{\mathrm{f}} \mathrm{S} / \mathrm{N} \sim 10 ; 90 \% \text { confi } \\
\text { Statistical only. } \\
{ }^{\mathrm{g}} \text { For sources at } 10 \sigma, \mathrm{i} \\
{ }^{\mathrm{h}} \text { At } 400 \mathrm{MeV} \text {. } \\
{ }^{\mathrm{i}} \text { FWHM. }\end{array}$ & $\begin{array}{l}\text { or (full width at zer } \\
6 \mathrm{~s} \text { at high Galactic } \\
\text { at } 400 \mathrm{MeV} \text {. } \\
\text { nce limit (c.1.) radi } \\
\text { uding statistics and }\end{array}$ & $\begin{array}{l}\text { ensity). } \\
\text { ude, on-axis. } \\
\text { t high Galactic latitude. } \\
\text { ematics. }\end{array}$ \\
\hline
\end{tabular}

\section{DATA REDUCTION AND ANALYSIS}

Level 1 AGILE-GRID data were analyzed using the AGILE Standard Analysis Pipeline. A first step aligns all data times to terrestrial time (TT) and performs preliminary calculations. In a second step, an ad hoc implementation of the Kalman Filter technique (A. Giuliani et al. 2008, in preparation) is used for track identification and event direction reconstruction in detector coordinates. Subsequently, a quality flag is assigned to each GRID event: G, P, S, and L, depending on whether it is recognized as a $\gamma$-ray event, a charged particle event, a single-track event, or of uncertain nature, respectively. Then, an AGILE log file is created, containing all the information relevant to the computation of the exposure and live time. A third step creates the AGILE event files, excluding events flagged as particles. This step also reconstructs the event direction in sky coordinates.

Once the above steps are completed, the AGILE Scientific Analysis Package can be run. Counts, exposure, and Galactic background $\gamma$-ray maps are created with a bin size of $0.5^{\circ} \times 0.5^{\circ}$, for $E \geq 100 \mathrm{MeV}$. To reduce the particle background contamination we selected only events flagged as confirmed $\gamma$-ray events (filtercode =5), while all events collected during the South Atlantic Anomaly (SAA) were rejected (phasecode=18). We also rejected all the $\gamma$-ray events whose reconstructed directions form angles with the satellite-Earth vector smaller than $80^{\circ}$ (albrad $=80$ ), reducing the $\gamma$-ray Earth albedo contamination by excluding regions within $\sim 10^{\circ}$ from the Earth limb. The most recent versions (BUILD-12) of the Calibration files, which will be publicly available at the ASDC site, and of the $\gamma$-ray diffuse emission model (Giuliani et al. 2004) were used.

Since a complete analysis of the in-flight calibration is not available yet, we adopted the following procedure for the $\gamma$ ray flux determination. We ran the AGILE maximum likelihood procedure (ALIKE) on the whole observing period, in order to obtain a value for the average flux. We ran ALIKE both on $3 \mathrm{C} 454.3$ and on a long ( 12 days) set of observations of the Vela pulsar taken at approximately $23^{\circ}$ off-axis. Since Monte Carlo simulations show that the effective area and the pointspread function are similar for off-axis angles in the $25^{\circ}-35^{\circ}$ range, we estimated the $3 \mathrm{C} 454.3$ flux according to

$$
F_{3 \mathrm{C} 454.3}^{\mathrm{est}}=\frac{F_{3 \mathrm{C} 454.3}^{\mathrm{ALIKE}}}{F_{\text {Vela }}^{\mathrm{ALIKE}}} \times F_{\text {Vela }}^{\text {3EG }},
$$

${ }^{18}$ See http://agile.asdc.asi.it. 


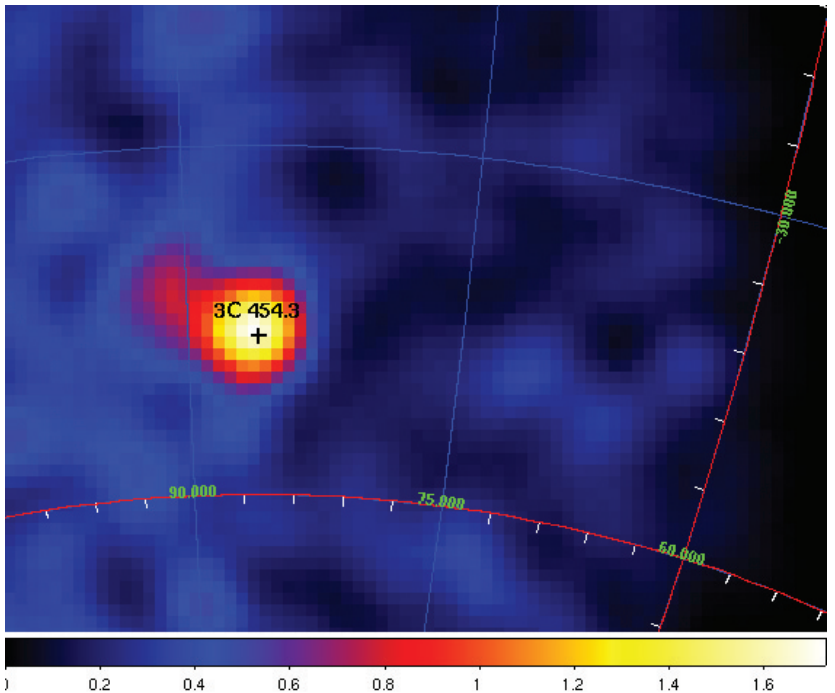

FIG. 1.-Gaussian-smoothed counts map $\left(238^{\circ} \times 28^{\circ}\right)$ in Galactic coordinates integrated over the whole observing period (2007 July 24 14:30 UT2007 July 30 11:40 UT). The cross is located at the $3 \mathrm{C} 454.3$ radio coordinates.

where the terms with the ALIKE superscript are derived by ALIKE, while those with the 3EG superscript come from the Third EGRET Catalog (Hartman et al. 1999).

Super-AGILE observed 3C 454.3 for a total on-source net exposure time of $250 \mathrm{ks}$. The source was observed at an offaxis position varying from $0.7^{\circ}$ to $2.5^{\circ}$ along the SA $X$-coordinate and from $-37.3^{\circ}$ to $-42.2^{\circ}$ along the $Z$-coordinate, due to the Sun constraints on the AGILE pointing. This implies that the source could be observed only by two of the four SA detectors, with imaging capability in only one direction $(Z)$ and an exposed area of about $1 / 8$ of the on-axis value (see Feroci et al. [2007] for a detailed description of the geometry of the FOV of the Super-AGILE detector).

\section{RESULTS}

Figure 1 shows a Gaussian-smoothed counts map $\left(\sim 38^{\circ} \times 28^{\circ}\right)$ in Galactic coordinates integrated over the whole observing period, using the selections described in $\S 4$. The source detection significance is $13.8 \sigma$ as derived from a maximum likelihood analysis. The $\gamma$-ray source detected by the AGILE-GRID is fully consistent

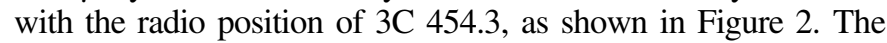
solid circle represents the AGILE error circle taking into account both statistic and systematic uncertainties, the dot-dashed circle represents the $95 \%$ maximum likelihood contour level, while the dotted circle is the Third EGRET Catalog $95 \%$ error circle (radius $0.28^{\circ}$ ). While the AGILE 95\% maximum likelihood contour level has a semimajor axis $a=0.33^{\circ}$ and a semiminor axis $b=$ $0.32^{\circ}$, the overall AGILE error circle has a radius $r=0.47^{\circ}$. The distance between the $3 \mathrm{C} 454.3$ radio position (square) and the AGILE 95\% maximum likelihood contour level barycenter ( $\operatorname{star} ; l=86.42^{\circ}, b=-38.37^{\circ}$ ) is $\sim 0.29^{\circ}$. We note that the EGRET $95 \%$ error circle corresponds to the sum of all the pointings toward the source (VP 1234; 1991 April-1995 October). During this period EGRET accumulated 467 counts above 100 $\mathrm{MeV}$, while AGILE, during its 1 week pointing, accumulated 107 counts above $100 \mathrm{MeV}$. The much larger period over which the EGRET source location was derived explains the relative sizes of the EGRET and AGILE error circles.

Figure $3 a$ shows the $\gamma$-ray light curve at 1 day resolution

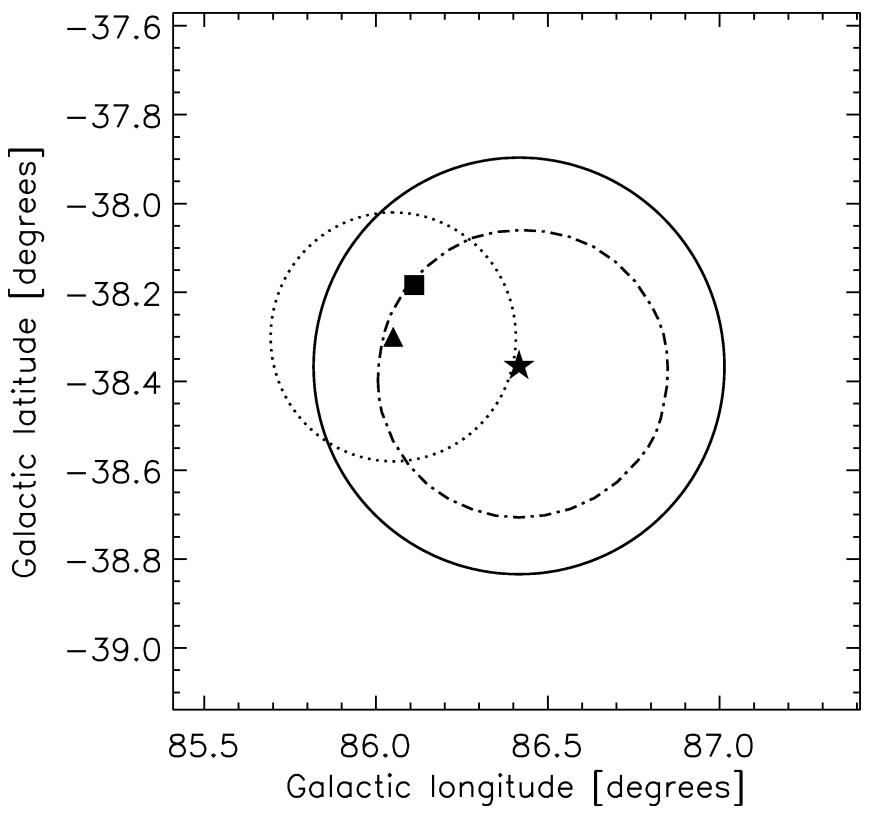

FIG. 2.-Dot-dashed circle: AGILE 95\% maximum likelihood contour level. Solid circle: AGILE 95\% error circle taking into account both statistic and systematic uncertainties. Dotted circle: EGRET 95\% error circle (Hartman et al. 1999). Star: AGILE 95\% maximum likelihood contour level barycenter. Triangle: EGRET $\gamma$-ray 3C 454.3 position (Hartman et al. 1999). Square: 3C 454.3 radio position.

for photons above $100 \mathrm{MeV}$. We note that 3C 454.3 is detected at a $4 \sigma$ level during almost the whole period on a 1 day timescale; this clearly indicates strong $\gamma$-ray flaring activity. The average $\gamma$-ray flux above $100 \mathrm{MeV}$ for the whole period is $F_{3 \mathrm{C} 454.3}^{\text {est }}=(280 \pm 40) \times 10^{-8}$ photons $\mathrm{cm}^{-2} \mathrm{~s}^{-1}$. The spectral analysis will be presented in a forthcoming paper.

The average $\gamma$-ray flux as well as the daily values of the six
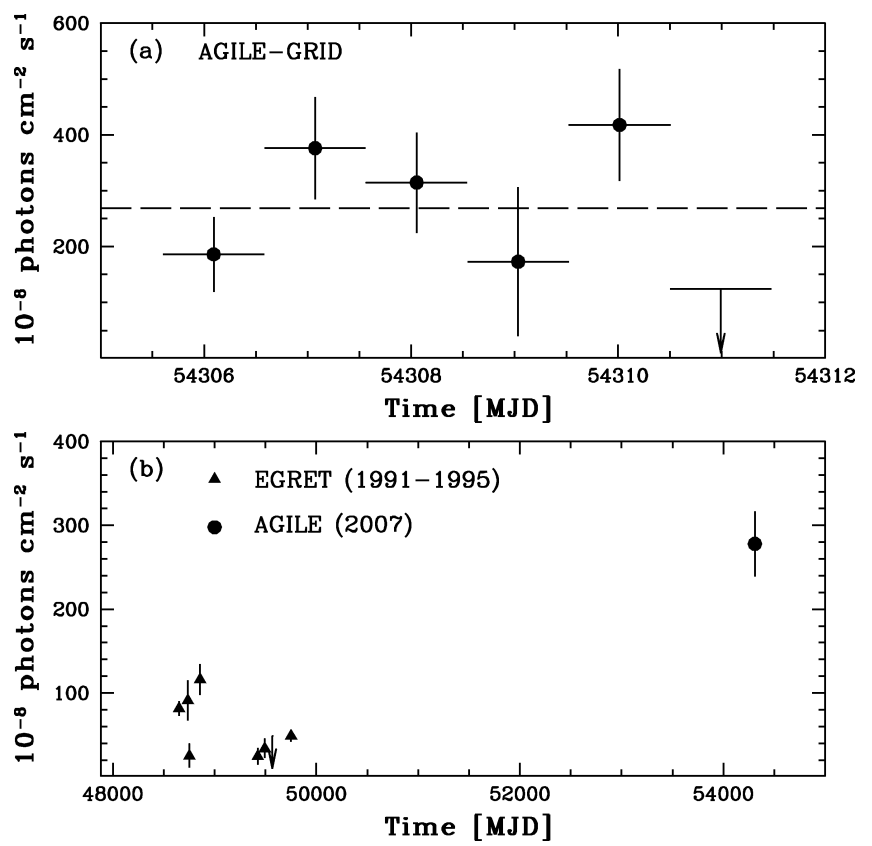

FIG. 3.-(a) AGILE-GRID $\gamma$-ray light curve at $\approx 1$ day resolution for $E>$ $100 \mathrm{MeV}$ in units of $10^{-8}$ photons $\mathrm{cm}^{-2} \mathrm{~s}^{-1}$. The downward arrow represents a $2 \sigma$ upper limit. The dashed line represents the weighted mean flux. (b): EGRET (triangles) and AGILE-GRID (circles) $\gamma$-ray light curve in units of $10^{-8}$ photons $\mathrm{cm}^{-2} \mathrm{~s}^{-1}$. EGRET data are from Hartman et al. (1999). 
days were derived according to Mattox et al. (1993). First, the entire period was analyzed to determine the diffuse gas parameters and then the source flux density was estimated independently for each of six 1 day periods with the diffuse parameters fixed at the values obtained in the first step. The average $\gamma$ ray flux above $100 \mathrm{MeV}$ for the whole period is the highest ever detected from this source, as shown in Figure $3 b$. Fitting the GRID fluxes to a constant model (the weighted mean of the 1 day average flux values) yields $\chi^{2}=2.611$ for 5 degrees of freedom (dof); therefore we can exclude that the fluxes are constant at the $97.7 \%(\sim 2.3 \sigma)$ level. A more robust assessment of the source behavior will be possible when the calibrations are finalized. However, an indication can be seen of a quick flux decay toward the end of the observation.

The source was not detected (above $5 \sigma$ ) by the Super-AGILE Iterative Removal Of Sources (IROS) applied to the $Z$ image, in the 20-60 keV energy range. An upper limit to the observed count rate was obtained by a study of the background fluctuations at the position of the source and a simulation of the source and background contributions with IROS. Assuming a power-law spectral shape with photon index $\Gamma=1.5$ (extrapolated from the spectral fit of the simultaneous Swift 0.3-10 $\mathrm{keV}$ data, whose analysis will be presented elsewhere), we find a $3 \sigma$ upper limit of $2.3 \times 10^{-3}$ photons $\mathrm{cm}^{-2} \mathrm{~s}^{-1}$ on the average flux from $3 \mathrm{C} 454.3$, allowing for a $50 \%$ systematic error to take into account the early status of the calibration of the SuperAGILE response.

\section{DISCUSSION}

In this Letter we presented the first blazar detection by AGILE. Previous EGRET observations and detections of 3C 454.3 were carried out in different observational conditions (off-axis angle between $7^{\circ}$ and $23^{\circ}$; typical viewing period duration 8 days, with a single long observation lasting approximately 19 days, $7^{\circ}$ offaxis). A significant $\gamma$-ray flare was detected in 1992 JanuaryFebruary with a peak level of $F_{E>100 \mathrm{MeV}}=140 \times 10^{-8}$ photons $\mathrm{cm}^{-2} \mathrm{~s}^{-1}$ followed by a 2 week decreasing trend (Hartman et al. 1993). Subsequent EGRET detections in 1992 April-May confirmed the flux level near $F_{E>100 \mathrm{MeV}}=100 \times 10^{-8}$ photons $\mathrm{cm}^{-2} \mathrm{~s}^{-1}$ while an observation during 1995 November-December detected a significantly lower flux, near $F_{E>100 \mathrm{MeV}}=$ (20-30) $\times 10^{-8}$ photons $\mathrm{cm}^{-2} \mathrm{~s}^{-1}$ (Aller et al. 1997).

The AGILE detection therefore shows the highest $\gamma$-ray flux ever detected from 3C 454.3. It is remarkable that a FSRQ such as $3 \mathrm{C} 454.3$ is quite active in the high-energy domain over a timescale of two decades. It is at the moment unclear what determines the jet activity and the high-energy emission from the environment close to the massive black hole of 3C 454.3. In the EGRET era, nine blazars were observed to reach a $\gamma$-ray flux (in units of $10^{-8}$ photons $\mathrm{cm}^{-2} \mathrm{~s}^{-1}$ above $100 \mathrm{MeV}$ ) between 100 and 200, two (3C 279 and 1622-297) between 200 and 300, and two $(0528+134$ and 1622-297) between 300 and 350 . Thus, our detection of 3C 454.3 is comparable to the highest $\gamma$-ray fluxes detected so far.

The AGILE $\gamma$-ray detection of 3C 454.3 supports the idea that a special class of blazars shows significant $\gamma$-ray activity on timescales of decades that clearly distinguishes them from other blazar candidates that share similar radio, optical, or X-ray properties, but show no $\gamma$-ray emission. What determines such behavior, observed in 3C 454.3, 3C 279 and similar $\gamma$-ray blazars, is currently unclear. Kellermann et al. (2004) find that radio jets of strong $\gamma$ ray blazars seem to have significantly faster median apparent speeds, $(8.6 \pm 1.6) c$, than blazars with no $\gamma$-ray emission, $(3.9 \pm 1.1) c$. This implies that $\gamma$-ray sources have larger Doppler factors than blazars with no $\gamma$-ray emission. Recently, Kovalev et al. (2005) found that $\gamma$-ray blazar radio jets appear to have more compact VLBA structure than blazars with no $\gamma$-ray emission, contrary to earlier results (Kellermann et al. 1998) wherein the authors reported no clear differences in the jet morphology between $\gamma$-ray-loud and $\gamma$-ray-quiet blazars. Because of its large field of view and relatively flat sensitivity in the $\gamma$-ray energy range, AGILE can, for the first time, simultaneously monitor a large number of known and candidate $\gamma$-ray blazars, both in $\gamma$-ray and in the hard X-ray energy bands. This will make it possible to study in detail the apparent dichotomy in the blazar population between $\gamma$-ray-loud and $\gamma$-ray-quiet sources. Moreover, blazar $\gamma$-ray light curves can now be monitored on timescales of the order of 3 weeks, allowing us to perform long-term variability studies both in the high-energy range and correlated with the optical band. This will be crucial in investigating correlations and time delays between optical flares and $\gamma$-ray emission.

We thank the referee for his/her very constructive comments. The AGILE mission is funded by the Italian Space Agency (ASI) with scientific and programmatic participation by the Italian Institute of Astrophysics (INAF) and the Italian Institute of Nuclear Physics (INFN). This investigation was carried out with partial support under ASI contract I/089/06/1. We warmly thank G. Tosti for his tireless monitoring efforts. We wish to express our gratitude to the Carlo Gavazzi Space, Thales Alenia Space, Telespazio, and ASDC/Dataspazio Teams that implemented the necessary procedures to carry out the AGILE repointing.

\section{Facilities: AGILE}

\section{REFERENCES}

Aller, M. F., et al. 1997, in AIP Conf. Proc. 410, Proc. Fourth Compton Symposium, ed. C. D. Dermer et al. (New York: AIP), 1423

Barbiellini, G., et al. 2001, in AIP Conf. Proc. 587, Gamma 2001: GammaRay Astrophysics, ed. S. Ritz et al. (New York: AIP), 754

Bulgarelli, A., et al. 2007, ATel, 1167, 1

Costa, E., et al. 2001, X-Ray Astronomy: Stellar Endpoints, AGN, and the Diffuse X-ray Background, ed. N. E. White et al. (New York: AIP), 582

Feroci, M., et al. 2007, Nucl. Instrum. Methods Phys. Res. A, 581, 728

Giommi, P., et al. 2006, A\&A, 456, 911

Giuliani, A., et al. 2004, Mem. Soc. Astron. Italiana Suppl., 5, 135

2006, Nucl. Instrum. Methods Phys. Res. A, 568, 692

Hartman, R. C., et al. 1992, IAU Circ., 5477, 2 1993, ApJ, 407, L41 1999, ApJS, 123, 79

Kellermann, K. I., et al. 1998, AJ, 115, 1295

-. 2004, ApJ, 609, 539
Kovalev, Y. Y., et al. 2005, AJ, 130, 2473

Labanti, C., et al. 2006, in Proc. SPIE, 6266, 62663Q

Mattox, J. R., et al. 1993, ApJ, 410, 609

Perotti, F., Fiorini, M., Incorvaia, S., Mattaini, E., \& Sant'Ambrogio, E. 2006,

Nucl. Instrum. Methods Phys. Res. A, 556, 228

Pian, E., et al. 2006, A\&A, 449, L21

Prest, M., Barbiellini, G., Bordignon, G., Fedel, G., Liello, F., Longo, F., Pontoni, C., \& Vallazza, E. 2003, Nucl. Instrum. Methods Phys. Res. A, 501, 280

Tavani, M., et al. 2006, in Proc. SPIE, 6266, 626603

Vercellone, S., Soldi, S., Chen, A. W., \& Tavani, M. 2004, MNRAS, 353, 890

Vercellone, S., et al. 2007, ATel, 1160, 1

Villata, M., et al. 2006, A\&A, 453, 817 2007, A\&A, 464, L5

von Montigny, C., et al. 1995, ApJ, 440, 525

Zhang, S., et al. 2002, A\&A, 386, 843 